\title{
Performance Improvement of IEEE 802.15.4 Beacon-Enabled WPAN with Superframe Adaptation Via Traffic Indication*
}

\author{
Zeeshan Hameed Mir ${ }^{1}$, Changsu Suh ${ }^{2}$, and Young-Bae Ko ${ }^{1}$ \\ ${ }^{1}$ College of Information \& Communication, Ajou University, Republic of Korea \\ ${ }^{2}$ R \& D Department, Hanback Electronic, Republic of Korea \\ zhmir@ajou.ac.kr, scs@hanback.co.kr, youngko@ajou.ac.kr
}

\begin{abstract}
The IEEE 802.15.4 standard provides the widely accepted solution for low-cost and low-power wireless communications. Despite its design support for low duty-cycle operation, the fixed superframe size in Beacon-enabled mode limits its capabilities due to two contrasting goals; energy efficiency and higher data throughput. In this paper, we propose an enhancement of IEEE 802.15.4 Beacon-enabled mode which adaptively adjusts the active period based on the traffic information. In order to detect the data traffic in the networks, the proposed scheme utilizes the IEEE 802.15.4 CCA function. Evaluation results show that our scheme can improve energy efficiency as well as data throughput.
\end{abstract}

\section{Introduction}

IEEE 802.15.4 WPAN (Wireless Personal Area Networks) standard [1] supports for low-cost and low-power wireless connectivity among resource-limited devices. Especially, IEEE 802.15.4 MAC achieves a low-duty cycle operation by means of its Beacon-enabled mode. In this mode, a PAN coordinator periodically disseminates a superframe structure bounded by a beacon frame into the network and manages its active/inactive period. Any associated devices are allowed to communicate in the active period and conserve energy by turning off their transceivers during the inactive period. However, a fixed duration of the active period limits its overall performance by two means: idle listening [2] and lower data throughput.

In this paper, we proposed a novel scheme for mitigating the idle listening problem and improving data throughput in the current IEEE 802.15.4 Beaconenabled mode. In our proposed scheme, a coordinator can adaptively adjust the active period based on the data traffic information of associated devices. When a data traffic load is low, the active period is reduced to conserve energy consumption regardless of a superframe duration. However, with a higher traffic

\footnotetext{
* This work was in part supported by the MIC (Ministry of Information and Communication), Korea, under the ITRC support program supervised by IITA, (IITA2006-C1090-0602-0011) and (IITA-2006-C1090-0603-0015).
} 
load, the active period becomes lengthen up to a total beacon interval to improve data throughput. For performance evaluation, we have implemented our proposed scheme as well as the IEEE 802.15.4 full-standard using the TOSSIM [3] simulator. Evaluation results show that the proposed scheme outperforms in terms of both energy efficiency and data throughput.

\section{The Proposed Scheme}

To reduce energy consumption but still improve data throughput, the proposed scheme adaptively adjusts the active period based on the data traffic information. In our description, we use the term sentinel duration to refer to a special epoch for detecting the traffic information.

\subsection{Adaptive Active Duration}

In our scheme, devices having no data traffic are not required to continuously maintain an active state even when they are in their superframe duration part. The sentinel duration is periodically performed with a length equivalent to a superframe duration in a single beacon interval as shown in Fig. 1. In the proposed scheme, the value for Superframe Order (SO) is set to be smaller than the Beacon Order (BO). Therefore, several occurrences of sentinel duration can be performed in one beacon interval. At the start of a sentinel duration, if a node finds pending data traffic in its queue buffer, it tries to convey the traffic information to its coordinator. On detecting traffic presence from devices at some sentinel duration, the coordinator maintains an active RF state to receive data frames until the next sentinel duration. If there is no data traffic at any sentinel duration, the coordinator and devices can enter or continuously maintain the sleep state accordingly. Devices can continuously carry out the sentinel duration so that the pending data traffics can be transmitted to the coordinator even after the superframe duration is over. In this way, data throughput can be increased while decreasing latency when the data traffic load is high.

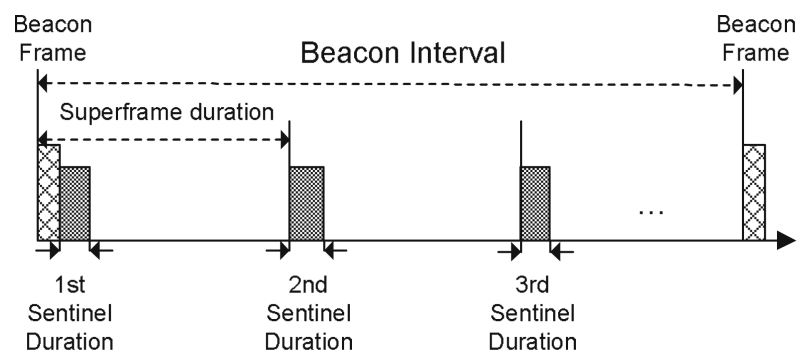

Fig. 1. Performing sentinel durations in a beacon interval 


\subsection{Traffic Indication Technique}

Our traffic indication technique utilizes the IEEE 802.15.4 CCA function. At the start of a sentinel duration, nodes having data traffic start to transmit it based on the slotted CSMA/CA similar to the original 802.15.4. In order to check the existences of data traffic, our traffic indication technique just waits for general packet frame's signal during the maximum contention period. The sentinel duration $\left(T_{-} S D\right)$ of traffic indication technique can be calculated as

$$
T \_S D=\left(2^{B E}-1\right) \times \text { aUintBackof f Period }
$$

where $B E$ means a back-off exponent value and its maximum value is 5 in the IEEE 802.15.4 standard, therefore $T_{-} S D$ is calculated as 620 symbols. As a transmitted packet should appear within the maximum 620 symbols, traffic indication technique can detect existence of the traffic information within this duration. If one of the CCA becomes busy during T_SD, traffic indication technique decides that there is data traffic. However, if no data is generated during $T_{-} S D$, nodes decide that there is no traffic in this active period and enter into the sleep mode. Since our traffic indication technique transmits no additional traffic indicator frames, it is consistent with the original IEEE 802.15.4 MAC without any conflict and control packet transmission overheads.

\section{Performance Evaluations}

We have evaluated the performance of proposed scheme and compared it with the original 802.15 .4 by using TOSSIM [3]. In our simulation model, we evaluated the performance of these two schemes in a star topology. One device is chosen as the coordinator while the other devices act as the associated devices (i.e., general devices). The data frame size is set to 30 bytes. The total simulation time is 200 seconds, and the energy consumption model is consistent to the one presented in [2]. We assume that all nodes are already associated with a coordinator, and only $20 \%$ of the nodes generate data frame every 1 second. We have simulated with the various number of devices which can influence of data traffic load. In these simulations, the values for $\mathrm{SO}$ and $\mathrm{BO}$ are 3 and 6 , respectively.

Fig. 2 plots the values for different performance matrices as a function of number of nodes. In terms of the aggregate throughput, our proposed scheme always performs better than the original 802.15.4, as shown in Fig. 2(a). Since the proposed scheme has an adaptive active duration based on the data traffic information, it is not limited by superframe duration, unlike the original 802.15.4. During the high data traffic load, the active duration lengthens as long as the Beacon Interval (BI), whereas in low data traffic scenarios it becomes as short as several sentinel durations. This observation can lead to the better performance of our schemes in terms of a delivery ratio as well. Fig. 2(b) shows the total energy consumption of the two protocols. Our proposed scheme can reduce the idle listening problem by adjusting the active duration. Therefore, $80 \%$ nodes having no data traffic can save energy in our scheme while nodes in the original 802.15.4 have to maintain active state for a full superframe duration. 

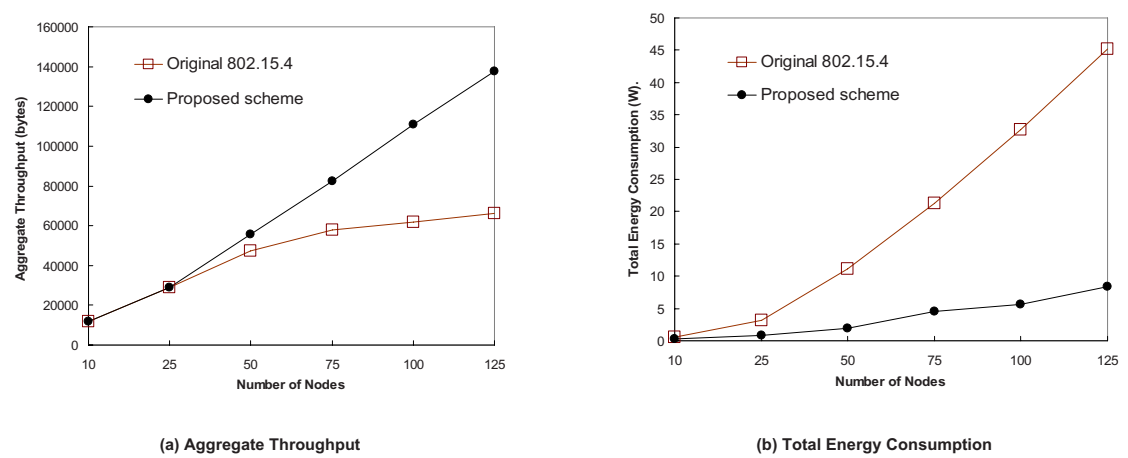

Fig. 2. Simulation results according to number of nodes with $\mathrm{SO}=3$ and $\mathrm{BO}=6$

\section{Conclusion}

In this paper, we propose the new IEEE 802.15.4 Beacon-enabled mode. We show that by utilizing available 'traffic information' based on our traffic indication technique help to adjust the active duration in order to conserve extra energy consumption and to achieve a higher throughput. The evaluation results compared with original IEEE 802.15.4 show that our proposed scheme outperforms in aspects of the energy consumption and throughput.

\section{References}

1. IEEE Std 802.15.4: Wireless Medium Access Control and Physical Layer specification for Low Rate Wireless Personal Area Networks, Dec. 2003.

2. W. Ye, J. Heidemann, and D. Estrin, "Medium Access Control with Coordinated Adaptive Sleeping for Wireless Sensor Networks," In IEEE Transaction on Networking, June 2004.

3. P. Levis, N. Lee, M. Welsh, and D. Culler, "TOSSIM: Accurate and Scalable Simulation of Entire TinyOS Applications", In ACM SenSys, Nov. 2003. 